\title{
Elevated Expression of ASXL2 is Associated with Poor Prognosis in Colorectal Cancer by Enhancing Tumorigenesis and Inducing Cell Proliferation
}

This article was published in the following Dove Press journal: Cancer Management and Research

\author{
Ran Cui ${ }^{1, *}$ \\ Ludi Yang ${ }^{2, *}$ \\ Yiwei Wang iD ${ }^{2, *}$ \\ Ming Zhong ${ }^{3}$ \\ Minhao $\mathrm{Yu}^{3}$ \\ Bo Chen' \\ 'Department of Hepatopancreatobiliary \\ Surgery, East Hospital Affiliated Tongji \\ University, Tongji University School of \\ Medicine, Shanghai 200I20, People's \\ Republic of China; ${ }^{2}$ State Key Laboratory \\ of Oncogenes and Related Genes, \\ Shanghai Cancer Institute, Shanghai Jiao \\ Tong University, Shanghai 200240, \\ People's Republic of China; ${ }^{3}$ Department \\ of Gastrointestinal Surgery, Ren Ji \\ Hospital, Shanghai Jiao Tong University \\ School of Medicine, Shanghai, 200I27, \\ People's Republic of China \\ *These authors contributed equally to \\ this work
}

Objective: Colorectal cancer is one of the most common malignant tumors worldwide. ASXL2 is an enhancer of the trithorax and polycomb genes, which have been proven to act in many tumor types. The role of ASXL2 in the occurrence and development of tumors has been extensively studied in recent years. However, the relationship between ASXL2 and the prognosis of CRC is still unclear.

Materials and Methods: In this study, quantitative real-time polymerase chain reaction (qRT-PCR), Western blot analysis and immunohistochemistry (IHC) were used to examine the expression of ASXL2 in CRC tissues. Cells were transfected with siRNAs or lentivirus to regulate the expression of ASXL2. The effects of ASXL2 on the proliferation of CRC cells were determined by CCK 8 assay.

Results: This study demonstrated that ASXL2 was significantly more highly expressed in CRC specimens than in normal adjacent tissues. The upregulation of ASXL2 was related to advanced clinical stage. Patients who exhibited high expression levels of ASXL2 had poorer overall survival, whereas those with low expression of ASXL2 survived longer. Multivariate Cox regression analysis revealed that ASXL2 expression could be considered an independent prognostic factor for CRC. Inhibition or overexpression of ASXL2 markedly influenced the proliferation of CRC cells.

Conclusion: These results showed that ASXL2 could induce cell proliferation, which was associated with poor prognosis of CRC patients, suggesting that ASXL2 might be a new therapeutic target for CRC.

Keywords: ASXL2, colorectal cancer, cell proliferation, prognosis marker

\section{Background}

Based on the latest statistics, colorectal cancer (CRC) is ranked as the third most common lethal malignancy and the second leading cause of cancer-related mortality globally. ${ }^{1}$ Although the diagnosis and treatment options for CRC have been improved, the disease outcomes have largely remained unsatisfactory, particularly in patients with lymph node or distant metastasis. ${ }^{2,3}$ Tumor invasion and metastasis are the major causes of mortality in colorectal cancer. ${ }^{4}$ Therefore, it is important to analyze the factors related to the metastasis and prognosis of colorectal cancer. As research progresses, several etiologic factors related to $\mathrm{CRC}$ progression have been reported. $^{5-8}$ Therefore, understanding the underlying molecular mechanisms involved in CRC progression and identifying novel biomarkers for evaluating prognosis is of great significance.

\footnotetext{
Correspondence: Bo Chen

Department of Hepatopancreatobiliary Surgery, East Hospital Affiliated Tongji University, Tongji University School of

People's Republic of China

Email chenbo7349@sohu.com

Minhao Yu

Department of Gastrointestinal Surgery, Ren Ji Hospital, Shanghai Jiao Tong University School of Medicine, 160 Pujian

Road, Shanghai, People's Republic of China

Email fishmeangood@163.com
} 
The additional sex combs-like 2 (ASXL2) gene, belonging to the ASXL gene family, is located on chromosome 2 p23.3 and has a length of $144,722 \mathrm{bp}$. It contains 12 exons and encodes a nuclear protein with a length of 1435 amino acids. Notably, as homologous genes, ASXL1 and ASXL2 are positive regulators of trithorax and Polycomb (ETP) genes, both encode essential transcriptional and epigenetic regulatory proteins involved in important developmental stages. ${ }^{9-13}$ Compared with that of ASXL1, ASXL2 function has been less studied. Studies have shown the potential roles of ASXL2 in cardiac function, adipogenesis and osteoclastogenesis. ${ }^{14,15}$ In addition, ASXL2 was found to be related to the occurrence and development of tumors by being involved in transcriptional activation and repression in a context-dependent manner. ${ }^{16,17}$ Park et al found that ASXL2 could potentially be used as a biomarker for predicting the disease outcomes of breast cancer patients. The gene has been shown to facilitate breast cancer cell proliferation by linking ER $\alpha$ to histone methylation. ${ }^{18}$ In addition, existing evidence indicates that ASXL2 is associated with poor prognosis in many solid tumors, but the function of ASXL2 in colon cancer has not been fully elucidated. ${ }^{19-21}$

In this study, we compared the transcription and expression of ASXL2 between CRC tissues and adjacent normal tissues to explore the relationship between ASXL2 expression and clinicopathological features of patients with colon cancer. Survival analysis was performed to explore whether ASXL2 is a critical factor in the prognosis of colon cancer. In addition, we performed in vitro experiments to further confirm the impact of ASXL2 expression on the proliferative ability of CRC cell lines. Ultimately, we conclude that ASXL2 is related to the poor prognosis of colorectal carcinoma via induction of cell proliferation.

\section{Materials and Methods}

\section{Tissue Samples}

All specimens were histopathologically confirmed by the pathologists and obtained with informed consent. The samples were taken from 182 cases of CRC tissues obtained from colorectal surgery at the Renji Hospital, Shanghai Jiao Tong University School of Medicine from April 2003 to November 2010. This study was approved by the Ethics Committee of Renji Hospital, School of Medicine, Shanghai Jiao Tong University (RA-2020001). The duration of the follow-up covered the period between the day of surgery to the death of a patient or the last day of follow-up. These tissues were made into the tissue microarrays (TMA). Furthermore, the specimens used for qRT-PCR were derived from 52 cases of CRC tissues and paired normal adjacent tissues obtained from the surgery at Renji Hospital from April 2017 to November 2018. None of the patients received radiotherapy or chemotherapy before surgery. The clinical stages and histology were categorized based on the American Joint Committee on Cancer (AJCC) guidelines. ${ }^{22} \mathrm{We}$ received signed informed consent from all patients.

\section{Gene Expression Assay}

Trizol reagent (Invitrogen, Carlsbad, CA, USA) was used to isolate total RNA from the tissues and cell lines according to the manufacturer's instructions. Next, the PrimeScript RT-PCR kit (Takara, Japan) was used for reverse transcription. We performed real-time quantitative PCR (RT-qPCR) using a 7500 real-time PCR system (Applied Biosystems, Inc. USA). The following primer sequences were used for ASXL2 detection: forward: 5'GGA AAA GGG ACG TAG GAA GAA G-3'; reverse: 5'ACT CAT GGG TGT ATT GGG GTA-3'. 18S RNA served as an internal control. The relative expression levels of mRNA were calculated using the $2^{-\Delta \mathrm{Ct}}$ method. RNA was examined by PCR array kit (Boster, Shanghai, China).

\section{Western Blot (WB)}

Treated cells were lysed in RIPA buffer containing protease inhibitor (Beyotime, Nanjing, China). The BCA Protein Assay kit (Beyotime, Nanjing, China) was used to determine protein concentrations. Transfer the protein to polyvinylidene difluoride membrane after electrophoresis. Blocked with milk, membranes were incubated with primary and secondary antibodies. Bands were detected by enhanced chemiluminescence (ECL) kit using the imageforming system of Amersham Imager 600 .

\section{Immunohistochemistry (IHC)}

Tissue microarray (TMA) of this study was constructed by Suzhou Xinxin. Immunohistochemical (IHC) staining was performed by the avidin-biotin-peroxidase method. We used immunohistochemistry to detect the expression of ASXL2 expression in CRC. Firstly, deparaffinized the slides, remove the endogenous peroxidase with a $3 \%$ hydrogen peroxide solution. Then after blocked with serum, the slides were incubated with anti-ASXL2 
antibody (1:200, Abcam, USA) overnight at $4^{\circ} \mathrm{C}$. Normal goat serum instead of primary antibody as a negative control. Finally, incubate with secondary antibody for 1 hour at room temperature, observe the color reaction of the immunohistochemical staining section under a microscope. The score of IHC was based on the intensity of staining and the percentage of positive cells. ${ }^{23}$ The intensity of staining can be divided into: negative: 0 ; weak staining: 1; moderate staining: 2; strong staining: 3, and the scoring standards of the percentage of positive cells was: $0-4$ (<5\%: $0 ; 5 \%-30 \%$ : $1 ; 30 \%-50 \%$ : $2 ; 51 \%$ $75 \%: 3 ;>75 \%: 4)$. The H-score is the product of the positive cell score and the percentage of positive cells, and the samples were divided into low and high expression groups based on whether the final score is 6 or more. The $\mathrm{H}$-score analysis was carried out independently by two experienced pathologists who were blinded to the final clinical diagnosis of all cases studied.

\section{Cell Culture and Transfection}

This study was approved by the Ethics Committee of Renji Hospital, School of Medicine, Shanghai Jiao Tong University (RA-2020-001). The human CRC cell lines (SW480, SW1116, Caco-2, HT29) were purchases from Shanghai Institute of Biochemistry and Cell Biology, Chinese Academy of Sciences (Shanghai, China), and maintained in a DMEM medium augmented with fetal bovine serum $(10 \%)$ and antibiotics $(1 \%)$ at $37^{\circ} \mathrm{C}$ in an incubator with $5 \%$ carbon dioxide. The transfections were performed by Lipofectamine 2000 (Invitrogen, USA) as per the manufacturer's instructions. The plasmids, used for constructing the ASXL2 over-expressing CRC cells, were procured from the Genearray Biotechnology (Shanghai, China). ASXL2 silencing CRC cells were constructed by transfecting with siASXL2 (GeneTech). The following primer sequences were used for negative si-RNA detection: 3'GCTTCAGGCTACGCATTTA-5'.

\section{Cell Proliferation Assay}

Proliferation was measured using CCK8 assay. Cells were seeded into 96-well plates for $24 \mathrm{~h}$. CCK8 $(10 \mu \mathrm{g} / \mathrm{mL})$ was added into the plates, and then the absorbances at $450 \mathrm{~nm}$ were recorded (Biotek Instruments Inc., Winooski, VT, USA). Replicate experiments were performed. Cell survival was then calculated.

\section{Statistical Analysis}

The analyses were performed using GraphPad Prism 8 and SPSS 22.0 software. We applied the Student's $t$-test to determine the difference in ASXL2 expression between $\mathrm{CRC}$ tissues and normal adjacent tissues. Chi-square test was used to analyze the relationship between ASXL2 expression and the clinicopathological features of CRC patients. The Kaplan-Meier method and Log rank test were employed to produce the survival curve and compare the survival rate between groups. Cox proportional hazard model was used to conduct univariate and multivariate analyses. Statistical significance was set at $\mathrm{P}<0.05$. The analyses were performed using SPSS 22.0 and GraphPad Prism 8 software.

\section{Results}

\section{ASXL2 Was Significantly Elevated in CRC Patients}

Based on the qRT-PCR assay, the levels of ASXL2 mRNA in CRC tissues were remarkably higher than those in normal adjacent tissues $(\mathrm{P}<0.001)$ (Figure $1 \mathrm{~A})$. We also investigated data from the TCGA database. Among 524 subjects, the expression level of ASXL2 was higher in those with tumors than in normal individuals, which agrees with our results (Figure 1C). Furthermore, with increasing tumor differentiation, ASXL2 levels gradually decreased (Figure 1B). We next performed IHC analysis to detect the expression of ASXL2 in TMAs. There were 182 cases of $\mathrm{CRC}$ and matched normal adjacent tissues in the TMAs. Based on the results, we divided the cases into two groups according to the median expression of ASXL2. ASXL2 was downregulated in 97 (53.3\%) CRC specimens and upregulated in the remaining 85 (46.7\%) samples (Figure 2).

\section{Relationship Between ASXL2 Expression and Clinicopathological Features of CRC Patients}

To assess the clinical significance of ASXL2, the chisquare test was used to analyze the correlation between the expression of ASXL2 and clinicopathological parameters in 182 CRC patients (Table 1). Overexpression of ASXL2 in CRC patients was significantly related to AJCC stage $(\mathrm{p}=0.020)$, $\mathrm{T}$ stage $(\mathrm{p}=0.038)$, and $\mathrm{N}$ stage $(\mathrm{p}=$ 0.022). No considerable relationship was observed between ASXL2 expression level and other features, 
A

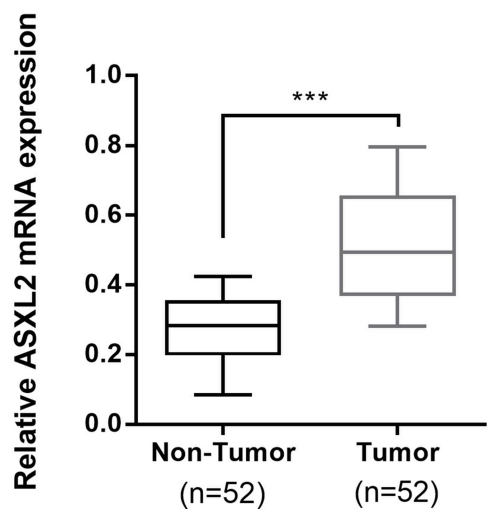

B

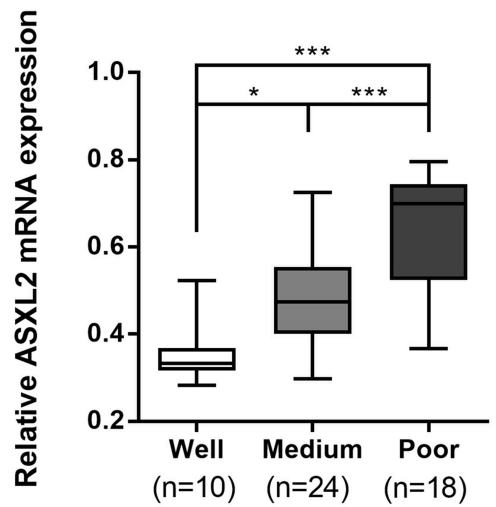

C

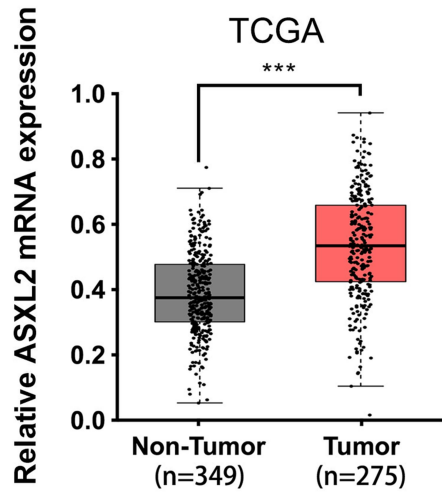

Figure I ASXL2 overexpression in CRC specimens detected by qRT-PCR analyse. (A) The mRNA levels of ASXL2 in I04 CRC tissues and paired normal mucosae were determined by qRT-PCR. (B) The mRNA levels of ASXL2 in CRC patients with different tumor differentiation were analyzed. (C) The mRNA levels of ASXL2 in 275 CRC tissues and 349 paired normal mucosae from TCGA database. ( $* \mathrm{P}<0.05$; ***P $<0.00 \mathrm{I})$.

\section{Colorectal cancer}
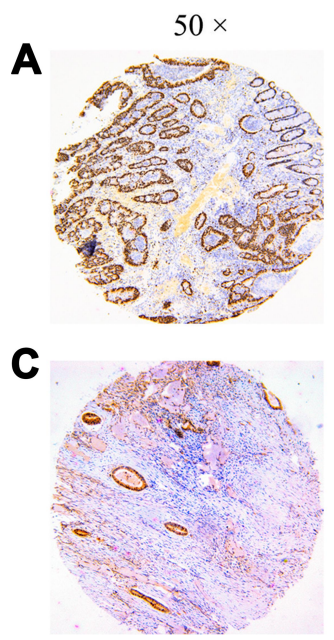

$400 \times$
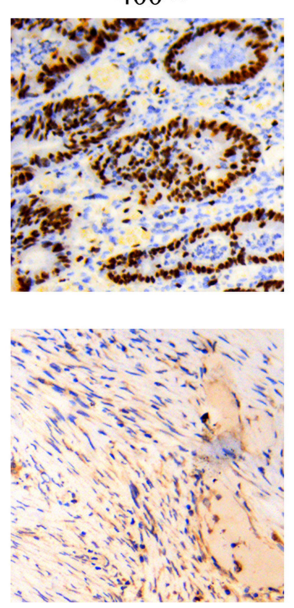

Normal colon mucosa

$50 \times$

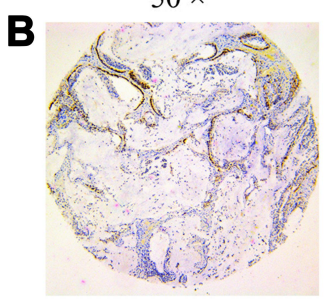

D

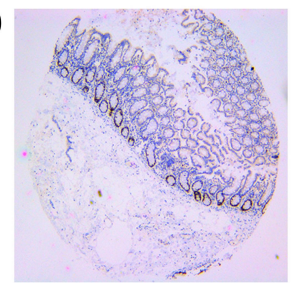

$400 \times$
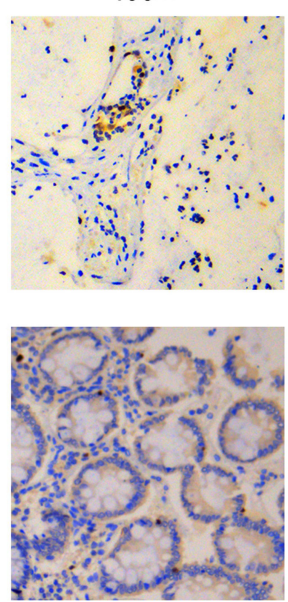

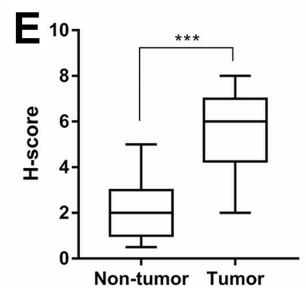

Figure 2 Representative immunohistochemistry staining of ASXL2 in CRC tissues and normal colon mucosae. (A and B) high expression of ASXL2. (C and D) low expression of ASXL2. Representative images are shown at 50x and 400x magnification respectively. H-score of Non-tumor and Tumor immunoreactivity (E). Data were presented as median (interquartile range). $(* * * \mathrm{P}<0.00 \mathrm{I})$.

including patient age, sex, tumor size and location and M stage $(P>0.05)$.

\section{ASXL2 Overexpression Was an Independent Risk Factor for Poor Prognosis in CRC}

Patients who had high ASXL2 expression exhibited a lower overall survival rate than patients with low expression of the gene (Figure $3 \mathrm{~A}, \log$ rank test, $\mathrm{P}<0.001$ ). Furthermore, the clinicopathological features of CRC patients were considered for the univariate analysis. The results suggested that the AJCC stage, TNM stage and expression of ASXL2 were risk factors for colorectal cancer prognosis. Additionally, the multivariate Cox regression analysis revealed that ASXL2 upregulation was an independent risk factor for poor colorectal cancer prognosis (Table 2).

In addition, the one-, three-, and five-year survival rates of patients with lymph node metastasis were lower than those in the non-lymph node metastasis group. Among those patients with lymph node metastasis, the five-year survival rate was the most significantly decreased. The subgroup analysis among the patients with lymphatic metastasis revealed that high ASXL2 expression was likely to confer a poor prognosis (Figure 
Table I Relationship Between ASXL2 Expression and Clinicopathological Features in 182 Colorectal Cancer Patients

\begin{tabular}{|c|c|c|c|}
\hline \multirow[t]{2}{*}{ Characteristics } & \multicolumn{2}{|c|}{ ASXL2 Expression } & \multirow[t]{2}{*}{ P-value } \\
\hline & Low $(\mathbf{N}=97)$ & High ( $\mathbf{N}=85$ ) & \\
\hline \multicolumn{4}{|l|}{ Age } \\
\hline$<65$ & 56 (59.6\%) & 38 (40.4\%) & 0.079 \\
\hline$\geq 65$ & $4 \mathrm{I}(46.6 \%)$ & 47 (53.4\%) & \\
\hline \multicolumn{4}{|l|}{ Gender } \\
\hline Male & 57 (50.4\%) & $56(49.6 \%)$ & 0.323 \\
\hline Female & 40 (58.0\%) & 29 (42.0\%) & \\
\hline \multicolumn{4}{|l|}{ Tumor size } \\
\hline$\leq 5 \mathrm{~cm}$ & 68 (55.7\%) & 54 (44.3\%) & 0.347 \\
\hline$>5 \mathrm{~cm}$ & $29(48.3 \%)$ & 31 (51.7\%) & \\
\hline \multicolumn{4}{|l|}{ Tumor location } \\
\hline Rectum & $72(53.3 \%)$ & $63(46.7 \%)$ & 1.000 \\
\hline Colon & $25(53.2 \%)$ & $22(46.8 \%)$ & \\
\hline \multicolumn{4}{|l|}{ AJCC Stage } \\
\hline 1 & 34 (70.8\%) & 14 (29.2\%) & 0.020 \\
\hline II & $23(52.2 \%)$ & $21(47.7 \%)$ & \\
\hline III & 31 (4I.9\%) & 43 (58.1\%) & \\
\hline IV & 9 (56.2\%) & 7 (43.8\%) & \\
\hline \multicolumn{4}{|l|}{$\mathrm{T}$ classification } \\
\hline TI & $16(76.2 \%)$ & $5(23.8 \%)$ & 0.038 \\
\hline $\mathrm{T} 2$ & $25(56.8 \%)$ & 19 (43.2\%) & \\
\hline T3 & $30(55.6 \%)$ & 24 (44.4\%) & \\
\hline $\mathrm{T} 4$ & 26 (4I.3\%) & 37 (58.7\%) & \\
\hline \multicolumn{4}{|l|}{$\mathrm{N}$ classification } \\
\hline No & 59 (62.8\%) & 35 (37.2\%) & 0.022 \\
\hline NI & $20(47.6 \%)$ & $22(52.4 \%)$ & \\
\hline N2 & $18(39.1 \%)$ & 28 (60.9\%) & \\
\hline \multicolumn{4}{|l|}{ M classification } \\
\hline Mo & 88 (53.0\%) & 78 (47.0\%) & 0.805 \\
\hline MI & 9 (56.2\%) & 7 (43.8\%) & \\
\hline
\end{tabular}

Note: The bold number represents the P-values with significant differences.

$3 \mathrm{~B}$ and $\mathrm{C}$ ). We also studied the data from the TCGA database. The higher ASXL2 expression and overall survival rate at mRNA levels showed a lower overall survival rate (Figure 3D).

\section{Effect of ASXL2 on CRC Cell}

\section{Proliferation in vitro}

To examine ASXL2 expression in vitro, the level of ASXL2 expression was assessed in human CRC cell lines (Caco-2, HT29, SW480, and SW1116) with different types of colorectal cancers (Figure 4A), and the cell lines were divided into two groups based on ASXL2 expression levels. Further experiments confirmed the degree of over/ underexpression of AXSL2 in Caco-2 and SW480 (Figure 4B).

Subsequently, Caco-2 and SW1116 cells were classified into the high expression group, whereas SW480 and HT29 cells were classified into the low expression group. We inhibited ASXL2 expression by transfecting siRNAASXL2 plasmids into the cell lines in the high expression group (Caco-2 and SW1116). Cell proliferation was considerably reduced with a decrease in ASXL2 expression (Figure 4C and D). Into the cell lines in the low expression group (HT29 and SW480), we transfected plasmids overexpressing ASXL2 to induce overexpression of ASXL2. Relative to the control group (empty vector), the group with overexpression of ASXL2 levels had a remarkable increase in cell proliferation (Figure 4E and F).

Additionally, we measured the Ki-67 level because it is known to be associated with cell proliferation. As we expected, the mRNA levels of Ki-67 were significantly increased with the overexpression of ASXL2 (and significantly decreased with knockdown of ASXL2), which confirmed our findings (Figure 4G and $\mathrm{H}$ ).

\section{Discussion}

CRC, like most forms of cancer, has biological and epidemiological heterogeneity, which have an impact on prognosis. ${ }^{24}$ In recent years, research has attempted to uncover how to improve the prognosis of patients with CRC. Therefore, understanding the underlying molecular mechanisms involved in the progression of $\mathrm{CRC}$ and identifying new biomarkers is of great importance for the prognostic evaluation and clinical management of CRC patients.

ASXL2, located on chromosome 20q11, is one of the enhancers of trithorax and polycomb genes. ${ }^{25}$ With the deeper study of tumors in recent years, ASXL2 has attracted much attention for its functional roles. Studies show that ASXL2 acts as an epigenetic regulator by recruiting a polycomb repressor complexes (PRCs), which activate DUBs and regulate cell proliferation. ${ }^{10}$ Recently, abnormal expression of ASXL2 has been reported in an increasing number of different types of tumors, and ASXL2 is known to cause poor prognosis in tumor patients. $^{9-11}$ However, the prognostic value and clinical significance of ASXL2 in CRC have not been reported to date.

Herein, we first revealed that ASXL2 was upregulated in CRC tissues relative to normal adjacent tissues 
A

Overall survival rate of different ASXL2 level

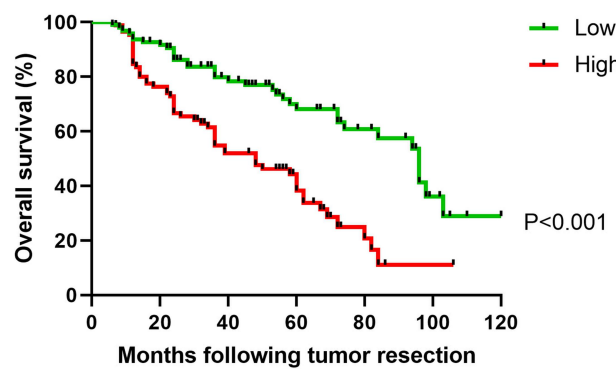

C

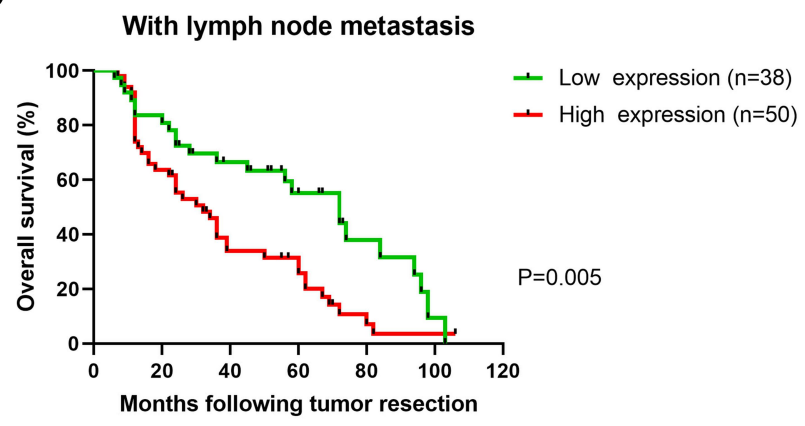

B Without lymph node metastasis

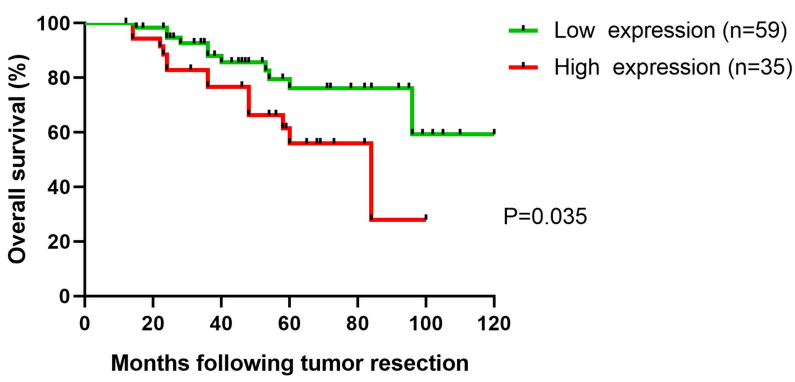

D

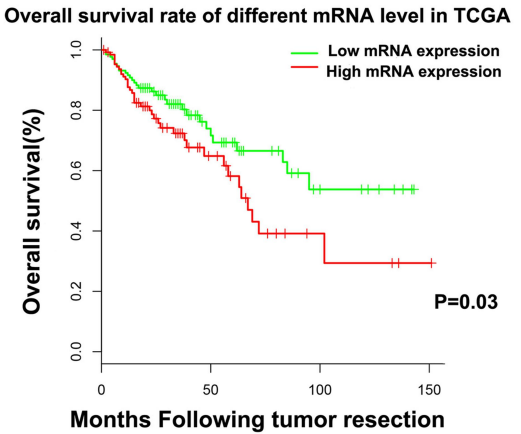

Figure 3 The prognostic significance of ASXL2 for CRC patients assessed via Kaplan-Meier analysis. The patients with lower ASXL2 expression had better OS (A). Significant difference was observed between high and low ASXL2 expression groups in both CRC patients with and without lymphatic metastasis (B and C). The patients with lower ASXL2 mRNA expression had better OS in TCGA database (D).

through RT-qPCR. In addition, we observed a remarkable decrease in the levels of ASXL2 mRNA in patients with low tumor differentiation and early clinical stages of $\mathrm{CRC}$, indicating that ASXL2 upregulation is related to an aggressive CRC phenotype. In addition, compared with those in the adjacent normal colonic mucosa, the mRNA levels of ASXL2 in CRC tissues were significantly overexpressed $(\mathrm{P}<0.001)$. Collectively, these findings suggest that ASXL2 may be an oncogene for CRC.
Subsequently, we examined the relationship between ASXL2 expression and clinicopathological features. We found that the upregulation of ASXL2 in CRC was significantly related to the AJCC stage, T stage, and $\mathrm{N}$ stage. The univariate analysis indicated that the overexpression of ASXL2 in CRC patients was closely related to OS. The multivariate analysis revealed that overexpression of ASXL2 in CRC patients was an independent predictor of poor disease outcomes. In general, these results indicate that ASXL2 expression can act as a biomarker for CRC

Table 2 Univariate and Multivariate Analyses Showing the Overall Survival in Colorectal Cancer

\begin{tabular}{|c|c|c|c|c|}
\hline \multirow[t]{2}{*}{ Variable } & \multicolumn{2}{|l|}{ Univariate } & \multicolumn{2}{|l|}{ Multivariate } \\
\hline & HR (95\% Cl) & $P$ value & HR (95\% Cl) & $P$ value \\
\hline ASXL2 & $2.782(1.791,4.321)$ & $<0.001$ & $2.150(1.350,3.423)$ & 0.001 \\
\hline Age & $0.920(0.608,1.391)$ & 0.692 & - & - \\
\hline Gender & I.I75(0.770, I.794) & 0.455 & - & - \\
\hline Size & $0.937(0.606, \mathrm{I} .446)$ & 0.768 & - & - \\
\hline Location & I.244(0.793, I.95I) & 0.343 & - & - \\
\hline AJCC Stage & $2.475(1.890,3.242)$ & $<0.001$ & - & - \\
\hline T classification & $2.405(1.820,3.178)$ & $<0.001$ & $2.075(1.431,3.009)$ & $<0.001$ \\
\hline $\mathrm{N}$ classification & $2.457(1.914,3.154)$ & $<0.001$ & $2.108(1.359,3.270)$ & 0.001 \\
\hline M classification & $4.548(2.432,8.505)$ & $<0.001$ & $5.932(1.928,18.252)$ & 0.002 \\
\hline
\end{tabular}

Note: The bold number represents the P-values with significant differences.

Abbreviations: $\mathrm{HR}$, hazard ratio; $\mathrm{Cl}$, confidence interval. 


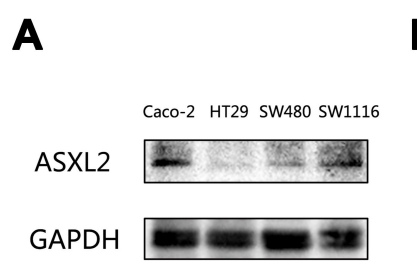

B
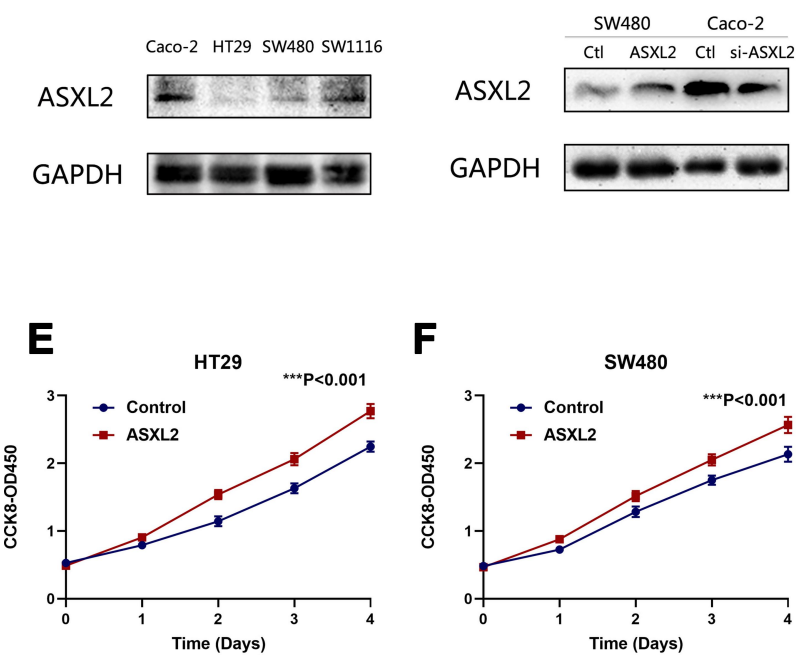
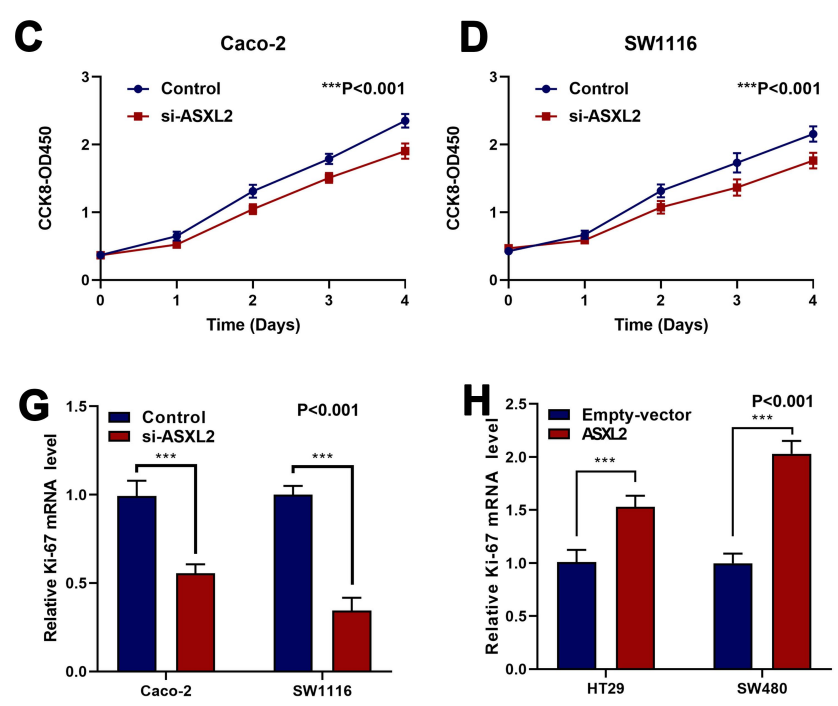

Figure 4 Effect of ASXL2 on CRC Cell Proliferation in Vitro. (A) The ASXL2 protein level in Caco-2, HT29, SW480 and SWIII 6 cells. (B) degree of over/underexpression of AXSL2 in Caco-2 and SW480. (C and D) siRNAs-ASXL2 decreased cell proliferation measured by CCK8 assays. (E and F) ASXL2 over-expressing plasmids increased cell proliferation measured by CCK8 assays. (G) Quantitative real-time PCR analysis of the mRNA levels of proliferation-associated gene (Ki-67) in cells with empty vectors and cells with siRNAs-ASXL2 plasmids in Caco-2, SWIII6. (H) Quantitative real-time PCR analysis of the mRNA levels of proliferation-associated gene (Ki-67) in cells with empty vectors and cells with ASXL2 over-expressing plasmids in $\mathrm{HT} 29$ and SW480. All data are representative of three independent experiments. (***P<0.00I)

and has the potential to become a new prognostic indicator for CRC patients.

Herein, we revealed that the levels of ASXL2 mRNA were higher in poorly differentiated CRC cell lines than in well-differentiated CRC cell lines. In addition, by overexpressing the ASXL2 gene in CRC cell lines, we found that overexpression of ASXL2 could significantly promote the proliferation of CRC cells. According to other literature, in a human breast cancer cell line, overexpression of ASXL2 was found to promote MCF7 cell proliferation and MCF7-derived tumor growth. ${ }^{9}$ The CCK-8 assay results also showed that low expression of ASXL2 resulted in a decrease in cell proliferation compared with that seen in the control group (empty vector). However, the exact molecular mechanism by which ASXL2 affects the growth and differentiation of colorectal cancer cells is still unknown.

In general, our data suggest that ASXL2 overexpression induces poor prognosis in colorectal carcinoma by enhancing cell proliferation. These results may offer new insights into the clinical management of $\mathrm{CRC}$ patients and the development of new therapeutic targets for CRC treatment. However, further studies on related signaling pathways are needed to fully elucidate the precise role of ASXL2 in CRC pathogenesis and how it can be exploited for the development of new therapeutic targets for CRC treatment.

\section{Conclusion}

Collectively, we elucidated the critical role of ASXL2 in human CRC progression, in which it was found to elevate proliferation. The discovery of the role of ASXL2 in CRC progression will aid in further CRC investigations and in developing therapeutic strategies against CRC.

\section{Data Sharing Statement}

All data generated or analysed during this study are included in this published article.

\section{Ethics Approval and Consent to Participate}

This research was approved by the Ethics Committee of Renji Hospital, School of Medicine, Shanghai Jiao Tong University (RA-2020-001), and informed consent was obtained from all patients before enrolling in the research program.

\section{Author Contributions}

All authors contributed to data analysis, drafting or revising the article, have agreed on the journal to which the article will be submitted, gave final approval of the version to be published, and agree to be accountable for all aspects of the work. 


\section{Funding}

This work was supported by the grant from the National Natural Science Foundation of China (No. 81702300), The Outstanding Clinical Discipline Project of Shanghai Pudong (No. PWYgy2018-02) and The Research project of Shanghai Municipal Health Commission (No. 20204Y0302).

\section{Disclosure}

The authors declare that they have no competing interests.

\section{References}

1. Siegel RL, Miller KD, Jemal A. Cancer statistics, 2020. CA Cancer $J$ Clin. 2020;70(1):7-30. doi:10.3322/caac.21590

2. Cunningham D, Atkin W, Lenz HJ, et al. Colorectal cancer. Lancet. 2010;375(9719):1030-1047. doi:10.1016/S0140-6736(10) 60353-4

3. Gallagher DJ, Kemeny N. Metastatic colorectal cancer: from improved survival to potential cure. Oncology. 2010;78(3-4):237-248. doi:10.1159/000315730

4. Jin C, Wang A, Chen J, Liu X, Wang G. Relationship between expression and prognostic ability of PTEN, STAT3 and VEGF-C in colorectal cancer. Exp Ther Med. 2012;4(4):633-639. doi:10.3892/ etm.2012.651

5. Faller WJ, Jackson TJ, Knight JR, et al. mTORC1-mediated translational elongation limits intestinal tumour initiation and growth. Nature. 2015;517(7535):497-500. doi:10.1038/nature13 896

6. Ma Y, Yang Y, Wang F, et al. Long non-coding RNA CCAL regulates colorectal cancer progression by activating Wnt/beta-catenin signalling pathway via suppression of activator protein 2alpha. Gut. 2016;65(9):1494-1504. doi:10.1136/gutjnl-2014-30 8392

7. Yue B, Liu C, Sun H, et al. A positive feed-forward loop between IncRNA-CYTOR and Wnt/beta-catenin signaling promotes metastasis of colon cancer. Mol Ther. 2018;26(5):1287-1298.

8. Liu C, Yue B, Yuan C, et al. Elevated expression of Thoc 1 is associated with aggressive phenotype and poor prognosis in colorectal cancer. Biochem Biophys Res Commun. 2015;468(1-2):53-58. doi:10.1016/j.bbrc.2015.10.166

9. Park UH, Kang MR, Kim EJ, et al. ASXL2 promotes proliferation of breast cancer cells by linking ERalpha to histone methylation. Oncogene. 2016;35(28):3742-3752. doi:10.1038/onc.2015.443

10. Daou S, Hammond-Martel I, Mashtalir N, et al. The BAP1/ASXL2 histone $\mathrm{H} 2 \mathrm{~A}$ deubiquitinase complex regulates cell proliferation and is disrupted in cancer. J Biol Chem. 2015;290(48):28643-28663. doi:10.1074/jbc.M115.661553
11. Jahn N, Agrawal M, Bullinger L, et al. Incidence and prognostic impact of ASXL2 mutations in adult acute myeloid leukemia patients with $\mathrm{t}(8 ; 21)$ (q22;q22): a study of the German-Austrian AML Study Group. Leukemia. 2017;31(4):1012-1015. doi:10.1038/leu.2017.18

12. Micol JB, Abdel-Wahab O. The role of additional sex combs-like proteins in cancer. Cold Spring Harb Perspect Med. 2016;6(10): a026526. doi:10.1101/cshperspect.a026526

13. Dinan AM, Atkins JF, Firth AE. ASXL gain-of-function truncation mutants: defective and dysregulated forms of a natural ribosomal frameshifting product? Biol Direct. 2017;12(1):24. doi:10.1186/ s13062-017-0195-0

14. Lai HL, Grachoff M, McGinley AL, et al. Maintenance of adult cardiac function requires the chromatin factor Asx12. $\mathrm{J} \mathrm{Mol} \mathrm{Cell}$ Cardiol. 2012;53(5):734-741. doi:10.1016/j.yjmcc.2012.08.014

15. Farber CR, Bennett BJ, Orozco L, et al. Mouse genome-wide association and systems genetics identify Asxl2 as a regulator of bone mineral density and osteoclastogenesis. PLoS Genet. 2011;7(4): e1002038. doi:10.1371/journal.pgen.1002038

16. Gildea JJ, Lopez R, Shearn A. A screen for new trithorax group genes identified little imaginal discs, the Drosophila melanogaster homologue of human retinoblastoma binding protein 2. Genetics. 2000;156(2):645-663.

17. Katoh M. Functional and cancer genomics of ASXL family members. Br J Cancer. 2013;109(2):299-306. doi:10.1038/bjc.2013.281

18. Park UH, Yoon SK, Park T, Kim EJ, Um SJ. Additional sex comb-like (ASXL) proteins 1 and 2 play opposite roles in adipogenesis via reciprocal regulation of peroxisome proliferator-activated receptor \{gamma\}. $J$ Biol Chem. 2011;286(2):1354-1363. doi:10.1074/jbc.M110.177816

19. Stephens PJ, Tarpey PS, Davies H, et al. The landscape of cancer genes and mutational processes in breast cancer. Nature. 2012;486 (7403):400-404. doi:10.1038/nature 11017

20. Grasso CS, Wu YM, Robinson DR, et al. The mutational landscape of lethal castration-resistant prostate cancer. Nature. 2012;487 (7406):239-243. doi:10.1038/nature11125

21. Jones S, Zhang X, Parsons DW, et al. Core signaling pathways in human pancreatic cancers revealed by global genomic analyses. Science. 2008;321(5897):1801-1806. doi:10.1126/science.1164368

22. Amin MB, Greene FL, Edge SB, et al. The eighth edition AJCC cancer staging manual: continuing to build a bridge from a population-based to a more "personalized" approach to cancer staging. CA Cancer J Clin. 2017;67(2):93-99. doi:10.3322/caac.21388

23. Yan DW, Li DW, Yang YX, et al. Ubiquitin D is correlated with colon cancer progression and predicts recurrence for stage II-III disease after curative surgery. Br J Cancer. 2010;103(7):961-969. doi:10.1038/sj.bjc.6605870

24. Kocarnik JM, Shiovitz S, Phipps AI. Molecular phenotypes of colorectal cancer and potential clinical applications. Gastroenterol Rep (Oxf). 2015;3(4):269-276.

25. Kennison JA. The Polycomb and trithorax group proteins of Drosophila: trans-regulators of homeotic gene function. Annu Rev Genet. 1995;29:289-303. doi:10.1146/annurev.ge.29.120195.001445

\section{Publish your work in this journal}

Cancer Management and Research is an international, peer-reviewed open access journal focusing on cancer research and the optimal use of preventative and integrated treatment interventions to achieve improved outcomes, enhanced survival and quality of life for the cancer patient.
The manuscript management system is completely online and includes a very quick and fair peer-review system, which is all easy to use. Visit http://www.dovepress.com/testimonials.php to read real quotes from published authors. 